\title{
Preference of Non-pharmaceutical Preoperative Anxiety Reduction Intervention in Patients Undergoing Elective Surgery
}

\author{
Sabo Saleh Dagona \\ Faculty of Health Studies, University of Bradford, \\ Richmond Road Bradford BD7 1DP, Bradford, United Kingdom \\ Uduak Archibong \\ Faculty of Health Studies, University of Bradford, \\ Richmond Road Bradford BD7 1DP, Bradford, United Kingdom \\ Gabrielle Tracy McClelland (PhD) \\ Faculty of Health Studies, University of Bradford, \\ Richmond Road Bradford BD7 1DP, Bradford, United Kingdom
}

\begin{abstract}
For patients to be treated, decisions about their care must be made before treatment begins. In case of pre-operative anxiety, it is currently unknown how clinicians and patients discuss information about the issue, and it is also not known whether clinicians consider (or are ready to consider) their patients' preferences of nonpharmaceutical pre-operative anxiety reduction interventions. At present no study has been conducted to find information on surgical patients' preferences of, and their involvement in decisions about non-pharmaceutical interventions for reducing their pre-operative anxiety. This paper investigates elective surgical patients' involvement in treatment decisions with the aim of finding out their preferred non-pharmaceutical pre-operative anxiety reduction interventions before they undergo elective surgery. Method: A survey method was used to collect data on patients' preference of nonpharmaceutical preoperative anxiety reduction interventions at a tertiary health facility in Nigeria. Participants: A sample of 30 participants-17 male and 13 female, schedule to undergo surgical operations was selected using a convenient sampling method. Their ages range between 17 to 70 years (mean age $=41.03$ and standard deviation = 16.09). Study design/procedure: To elicit preference of interventions, the study participants were presented with cards that contain picture of surgical patient receiving one of the non-pharmaceutical interventions used in reducing pre-operative anxiety. The pictures were presented one at a time for 30-40 seconds. The researcher then gives the participants a sheet of paper with the different interventions boldly written for the participants to rank order them according to the degree of their preferences. Through this process, data was collected from all the $\mathbf{3 0}$ participants. Results: The results obtained were entered into SPSS for analysis. Descriptive statistics, at $95 \%$ confidence was calculated to estimate the percentage, mean, standard deviation and confidence intervals based on the participants' preference of the interventions. Discussions: The findings were discussed alongside the existing literature and recommendations were offered for clinical practice and further research.
\end{abstract}

\section{INTRODUCTION}

It is obvious that, for any patient to be treated, some decisions about their care have to be made before the actual treatment. In case of pre-operative anxiety, however, it is currently unknown how clinicians and patients discuss and exchange relevant information about the health issue, and it is also not known whether clinicians consider (or are ready to consider) their patients' preferences of non-pharmaceutical pre-operative anxiety reduction 
interventions. Given the fact that pre-operative anxiety is not an illness per se for which the surgery is planned, but a problem that emerge as a result of the patients' appraisal of the procedure they are about to undergo which can affect the entire surgical process and reduce the patients' satisfaction with the surgery, discussing how best to treat it is very important. At present no study has been conducted to find information on surgical patients' preferences of, and their involvement in decision making about non-pharmaceutical interventions for reducing their pre-operative anxiety. This paper investigates elective surgical patients' involvement in treatment decisions with the aim of finding out their preferred nonpharmaceutical pre-operative anxiety reduction interventions before they undergo elective surgery.

\section{RESEARCH QUESTION}

What non-pharmaceutical pre-operative anxiety reduction intervention would elective surgical patients prefer to be used in reducing their pre-operative anxiety prior to elective surgery?

Are there gender differences in preference of non-pharmaceutical pre-operative anxiety reduction interventions among elective surgical patients?

\section{LITERATURE REVIEW}

Patients preference for intervention is about what patients actually want to happen or assume is going to happen if given their preferred treatment (Swift, Callahan, \& Vollmer, 2011). Treatment preference is about patients indicating the type of medication or intervention will be administered to them. It is therefore about, for example, choosing a behavioural instead of supportive intervention or preferring psychotherapy in place of pharmaceutical intervention etc. (McHugh, Whitton, Peckham, Welge, \& Otto, 2013). In this process, patients appraise the benefits, problems and inconveniences as they consider alternative interventions (Pomey, Ghadiri, Karazivan, Fernandez, \& Clavel, 2015). Hence, treatment preference is a subjective, personal assessment of how patients think through the likely effects of treatment on their health. Choices about treatments are not easy as there is no one treatment that is best for all patients. Instead, the best choice is subject to the individual patient's beliefs, experience and priorities.

Understanding patients' preferences of interventions are important in clinical practice, the benefits of which are gradually being recognised by healthcare professionals, as well as investigators involved in treatment outcome research (Coulter, Parsons, \& Askham, 2008). Involving patients in treatment decisions can improve satisfaction with treatment outcomes (Vahdat, Hamzehgardeshi, Hessam, and Hamzehgardeshi 2014), enable clinician-patient cooperative clinical relations and contribute in the adherence to interventions as patients feel that the intervention was mutually agreed upon by themselves and the clinicians (Coulter et al., 2008; Mitchell et al., 2012).

Because there are varieties of treatments for almost all illnesses, giving patients the opportunity to decide on and select their preferred intervention is widely advocated (Collins, Britten, \& Ruusuvuori, 2007; Coulter, 2011). People are naturally different, and their differences reflect the way they process information, react to and cope with illnesses and comorbidities, as well as psychosocial factors related to their health. Hence, an effective treatment for one patient might not work or be effective for another. It then follows that an effective pharmacological treatment could be the best medication of choice for one patient, a different patient can take the same treatment but will not, and yet another patient could not take the treatment even if needed (Montori, Brito, and Murad (2013). Therefore, attention must be paid to the biopsychosocial factors of the patients while considering what treatment is to be administered to them. 
The choice of treatment for patients requires not only the clinicians' expertise but also their practical wisdom to take some inputs from the patients (Montori et al., 2013). The traditional paternalistic approach that doctors, nurses or other clinicians know everything and therefore make decisions on their patients' behalf is today seen as an outmoded style of medical care (Tariman, Berry, Cochrane, Doorenbos, \& Schepp, 2012). In modern practice, patients' perceptions of their illness and how they want to be treated are important things to be considered by clinicians in planning treatments (Coulter, 2011; Coulter et al., 2008). Effective clinician-patient collaboration in treatment choice is highly advocated so that the two parties can work together to discuss the clinical and psychosocial aspects of the illness and the outcome of the treatment. The provision of patient care that respectfully and responsively considers patients' preferences, requirements, values and beliefs are at the centre of the widely advocated principles of evidence-based practice (Siminoff, 2013). When patients participate in treatment decision-making and their preferences considered, they feel more involved and empowered. According to Vahdat et al. (2014), involving patients in their healthcare decisions improves their knowledge, lowers their anxiety and increases their satisfaction with treatment; side-lining them in decisions about their treatment increases their dissatisfaction with healthcare (Coulter et al., 2008). Clinicians and patients are encouraged to work together to identify suitable therapeutic plans and select the ones preferred by the patients. However, this can only be achieved if patients are given the opportunity to be involved in decision making and are shown alternative interventions to choose from. This, according to (Vahdat et al., 2014), is an important step in ensuring a quality service characteristic of evidence-based practice.

\section{METHODS OF ELICITING PREFERENCE}

There are various ways of eliciting patients' preferences of intervention. The most popularly used method is to ask the patients directly what type of treatment they would prefer in treating their illness. This method entails asking the patients to state if they prefer medication, psychotherapy or a mixture of both treatments, or if they would prefer to be treated by a male or female clinician (Kocsis et al., 2009). This can be achieved through a simple discussion of preferences between the patients and the clinicians. Clinicians' understanding of their patients' treatment preferences is crucial for better communication to permit informed decisions that consider both the cost and benefits of the preferred treatment (Weernink et al., 2014). When considering treatment plans that involve choices between possibly devastating side effects and adverse outcomes, clinicians can have a greater role to play through good communication to guide their patients to make informed choices (Ha \& Longnecker, 2010). Therefore, clinicians should understand that patient preferences are strengthened by their personal beliefs and factors such as their age, gender and previous and current health states. This understanding will provide a basis for identifying potentially inaccurate beliefs which will form the basis of communication to facilitate choices and preferences in line with the patients' values (Gutknecht, Schaarschmidt, Herrlein, Augustin, \& Venereology, 2016). Ali and Ronaldson (2012) reported that when patients are given the opportunity to choose between two or more treatment plans that differ in effectiveness, mode of administration and risk of side effects, they choose the treatment that offers them the greatest value. The preferred choice made can tell about the value they attach to their chosen alternative. According to Ali and Ronaldson (2012), there are two major utility-based preference elicitation methods - stated preference elicitation method and revealed preference elicitation method.

(a) Stated preference elicitation method: this involves asking the patients to choose their preference from a group of two or more treatment options in an imaginary situation. The patients, therefore, make some sort of trade-offs based on the value (or utility) they attach to their chosen treatment Ali and Ronaldson (2012). This method of preference elicitation is 
important when a service or policy is not available at present, or where the goal is to assess patients' preferences among available treatment choices. The stated preference elicitation method is categorised into two:

(i) Cardinal Preference Method: in this method, patients' preferences for treatments are quantified by the responses they provide. The patients estimate the extent to which one treatment modality or health state is preferred over another (Ratcliffe, Brazier, Tsuchiya, Symonds, \& Brown, 2009). Ali and Ronaldson (2012) mentioned that the two most used cardinal preference methods include the standard gamble (SG) and time trade-off (TTO). While using these methods, the patients' trade-off between probabilities, uncertainties or risks related to a particular health state or service. These methods are used to scale patients' health states by requesting patients to a trade-off, for example, between years of good health (TTO) or threat of instant death (SG) against continuing in a specific health state for a given period. Cardinal preference methods are therefore used to come up with quantitative weightings for health states or values of treatment options Ali and Ronaldson (2012).

(ii) Ordinal Preference Elicitation Method: this method deals with ordering preference of two or more interventions without directly liking one alternative over the other (Ali \& Ronaldson, 2012). The most commonly used ordinal preference methods in the healthcare system are discrete choice experiments (DCE) and ranking exercises (de Bekker-Grob, Ryan, \& Gerard, 2012; Ratcliffe et al., 2009).

In DCE, patients are presented with a number of treatment options and then asked to select one. The ranking is a simple process, as it involves arranging items in a logical manner. The high relationship between rank responses and important valuations means that ranking may be used more generally to infer values of health states based on patients' preferences to be utilised in designing or administering interventions. The methods have sometimes been used to value health states, but are more commonly used to evaluate healthcare services, products, practices, interventions and policies (de Bekker-Grob et al., 2012).

(b) Revealed preference elicitation method: this method consists of the valuation of choices by assessing the actual patients' "behaviour in real-life situations so as to reveal the trade-offs they truly made" (Ryan \& Farrar, 2000). However, Ali and Ronaldson (2012) cautioned that conducting an assessment of patients' preferences using the revealed preference method is limited in that there must be existing health-related programmes or interventions for the patients to choose from. Also, the revealed preference method is not convenient for assessing assumed situations or interventions that are not yet developed. Based on these shortcomings, stated preference methods had become the most widely used method of eliciting preference in the fields of healthcare research and practice (Ali \& Ronaldson, 2012; Ryan \& Farrar, 2000).

In this study, elective surgical patients' preferences of non-pharmaceutical intervention for reducing pre-operative anxiety will be elicited through stated preference, using the ranking method.

\section{Study design}

\section{METHOD}

This study consists of presenting cards that contain a picture of a surgical patient receiving one of the non-pharmaceutical intervention approaches used in reducing pre-operative anxiety before undergoing a surgical operation. Participants were required to rank order the interventions according to the degree of their preferences. Ali and Ronaldson (2012) stated that the ranking exercise is undertaken to allow participants to rank order all available options from the most preferred to the least preferred alternative, thereby providing comprehensive 
ordering of all alternative choices. The research participants in this study were therefore instructed to rank order the non-pharmaceutical preoperative anxiety reduction interventions

(Music therapy, information and education, counselling services and video-film intervention), by giving the most preferred intervention the rank of 1 ; the next preferred to be given the rank of 2, and the least preferred the rank of 4. Fok, Paap, and Van Dijk (2012) claim that a complete ranking exercise may produce a more effective preference valuation as opposed to other methods, such as discrete choice experiments (DCEs), but they warned that the sorting task might be cognitively too complex or time-consuming for participants which may lead to biased estimation of preference.

\section{Participants}

A sample of 30 participants, consisting of 17 male and 13 female, diagnosed with different illnesses that call for surgical operation, were selected using a convenient sampling method. The participants' ages range between 17 to 70 years (mean age $=41.03$ and standard deviation = 16.09). Eight were single, 19 married and the remaining three were either divorced or separated. Almost half of the participants (14) were unemployed, 15 employed and one was retired. Their educational backgrounds varied; 1 participant was a student, 2 had primary education, 6 had secondary education, and 9 had tertiary education. On the other hand, a significant number of the participants (12) had informal education (either adult education or Qur'anic education).

\section{Procedure for data collection}

Patients' preferred non-pharmaceutical intervention for reducing pre-operative anxiety was elicited via a systematic approach informed by the work of (Miranda, 2009). Using this procedure, in a one-to-one meeting with the participants, the researcher presented the different non-pharmaceutical interventions used to reduce pre-operative anxiety in surgical patients. The interventions are those proved to be effective based on existing literature evidence and are the most frequently used in the hospital where the study was conducted. The researcher gives the participants a brief description of each intervention which has been printed in the form of an information leaflet. Participants who could not read had the description read to them by the researcher. The researcher then presented cards to the participants, one after the other, containing pictures of patients being administered the interventions. The cards were presented for 30-45 seconds. The researcher asked the participants to think about whether each intervention was suitable, appropriate and acceptable to them and therefore preferred to be used in reducing their pre-operative anxiety. Finally, a paper containing a list of the interventions was given to each participant to rankorder them according to their preferences. The most preferred intervention was given the rank of 1 ; the next least preferred the rank of 2 , whereas the least preferred intervention was given the rank of 4 .

\section{RESULTS \\ Preference of non-pharmaceutical interventions}

The participants' rankings of the different non-pharmaceutical interventions were analysed using SPSS software, version 23. Summary tables were constructed to present the scores of the participants showing the mean, standard deviation and confidence intervals to depict the degree of their preference of the various on-pharmaceutical preoperative anxiety reduction interventions. 
Table 1 Summary of participants' rankings for counselling services as a means of reducing preoperative anxiety

\begin{tabular}{|c|c|c|c|}
\hline Preference & Participants & Mean (SD) & 95\% Confidence interval \\
\hline Totally not preferred & 8 & $2.37(1.09)$ & $1.45-3.29$ \\
\hline Not preferred & 9 & $2.37(1.09)$ & $1.53-3.21$ \\
\hline Preferred & 7 & $2.37(1.09)$ & $1.36-3.39$ \\
\hline Most preferred & 6 & $2.37(1.09)$ & $1.22-3.52$ \\
\hline Total & 30 & - & - \\
\hline
\end{tabular}

The participants' scores for the preference of counselling services as a means of reducing preoperative anxiety show that, of the 30 study participants, eight $(26.7 \%, \mathrm{M}=2.37, \mathrm{SD}=1.09$; $95 \% \mathrm{CI}=1.45$ to 3.29 ) completely did not prefer counselling as an intervention for reducing their anxiety before surgery. Also, nine participants $(30 \%, \mathrm{M}=2.37, \mathrm{SD}=1.09 ; 95 \% \mathrm{CI}=1.53$ to 3.21 ) reported they did not prefer counselling; on the other hand, seven $(23.3 \%, \mathrm{M}=2.37, \mathrm{SD}=$ $1.09,95 \% \mathrm{CI}=1.35$ to 3.39$)$ and another $\operatorname{six}(20.0 \%)$ participants $(\mathrm{M}=2.37, \mathrm{SD}=1.09,95 \% \mathrm{CI}=$ 1.22 to 3.52 ) ranked counselling services as their preferred and most preferred pre-operative anxiety reduction interventions respectively. Figure 1 presents the participants' levels of preference for counselling services as an intervention for reducing their pre-operative anxiety.

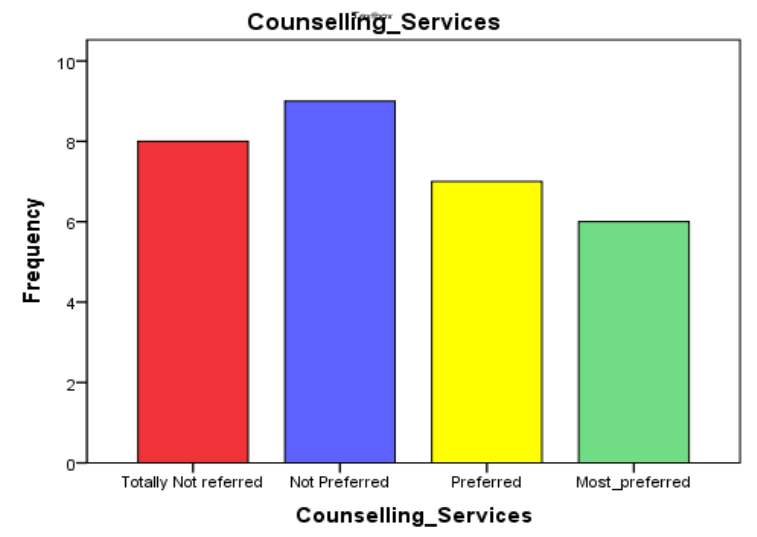

Figure 1 Bar graph for participants' preferences for counselling services as a means of reducing pre-operative anxiety

Table 2 Summary of participants' rankings for information and education intervention as a means of reducing pre-operative anxiety

\begin{tabular}{|l|c|c|c|}
\hline Preference levels & Participants & Mean (SD) & 95\% Confidence Interval \\
\hline Totally not preferred & 4 & $2.80(1.00)$ & 1.82 to 3.78 \\
Not preferred & 6 & $2.80(1.00)$ & 2.00 to 3.60 \\
Preferred & 12 & $2.80(1.00)$ & 2.23 to 3.37 \\
Most preferred & 8 & $2.80(1.00)$ & 2.11 to 3.49 \\
Total & 30 & & \\
\hline
\end{tabular}

On information and education intervention, the research participants' ranking scores indicate that only four (13\%, M $=2.8,95 \%$ CI [1.82 to 3.78]) of the participants indicated that they 'totally did not prefer' information and education as a strategy for reducing their pre-operative anxiety. Six other participants (20\%, M = 2.8, 95\% CI [2 to 3.6]) ranked information and education as a 'not preferred' choice, while a total of 12 participants $(40 \%, \mathrm{M}=2.8,95 \% \mathrm{CI}$ $[2.234,3.366])$ scored 'preferred', information and education, and the remaining eight (26.70\%, M = 2.8, 95\% CI [2.107, 3.493]) participants ranked information and education as their 'most preferred 'intervention for reducing their pre-operative anxiety before undergoing a surgical operation. In total, on the information and education sub-scale, $33.3 \%$ of the 
participants did 'not totally prefer' or 'did not prefer' information and education as a means of reducing their anxiety prior to surgery. On the other hand, more than half $(66.7 \%)$ of the participants either 'preferred', or 'most preferred' information and education as their chosen intervention for reducing pre-operative anxiety before undergoing surgery. The information for the participants' ranking of information and education as a strategy for reducing preoperative anxiety is presented in figure 2 below.

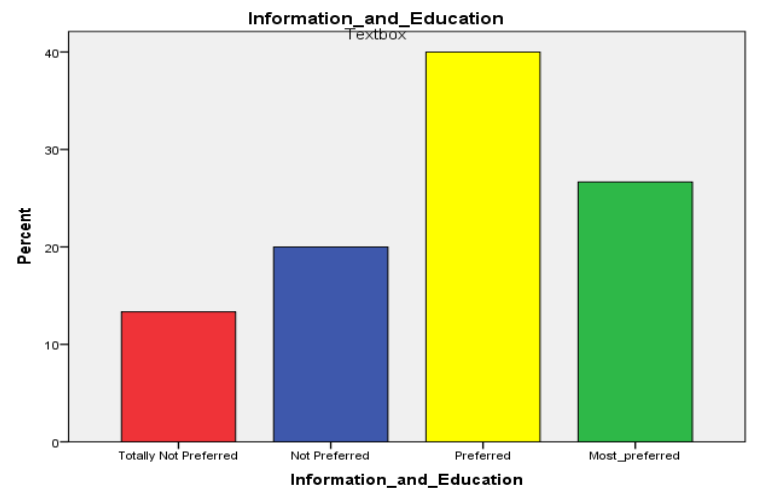

Figure 2: bar graph for participants' preference for information and education as a means of reducing pre-operative anxiety

Table 3 Summary of participants' rankings for counselling services as a means of reducing preoperative anxiety

\begin{tabular}{|l|c|c|c|}
\hline & Participants & Mean (SD) & 95\% Confidence Interval \\
\hline Totally not preferred & 17 & $1.8(1.0)$ & 1.325 to 2.275 \\
\hline Not preferred & 6 & $1.8(1.0)$ & 1.00 to 2.6 \\
\hline Preferred & 4 & $1.8(1.0)$ & 0.82 to 2.78 \\
\hline Most preferred & 3 & $1.8(1.0)$ & \\
\hline Total & 30 & & \\
\hline
\end{tabular}

The participants' rankings of music intervention show that, out of the 30 participants, more than half of the participants 17 (56.7\%, M = 1.8, 95\% CI [1.33 to 2.28]) 'totally did not prefer' music intervention as a means of reducing their anxiety. Another six $(20 \%, \mathrm{M}=1.8,95 \% \mathrm{CI}$ [12.6]) ranked music intervention as 'not preferred' pre-operative anxiety reduction intervention. On the other hand, four participants $(13.3 \%, \mathrm{M}=1.8,95 \% \mathrm{CI}$ [0.82 to 2.78]) preferred counselling, while the remaining three $(10 \%, \mathrm{M}=1.8-95 \% \mathrm{CI}$ [0.67-2.93]) participants indicated that music intervention is their 'most preferred intervention'. Thus, regarding music therapy, only $23.3 \%$ of the participants expressed a preference for music to be used in reducing their pre-operative anxiety. The large majority of the participants, $76.7 \%$, do not like music as an intervention to be used in reducing their anxiety prior to surgery. Figure 3 presents the participants' rankings of music intervention 


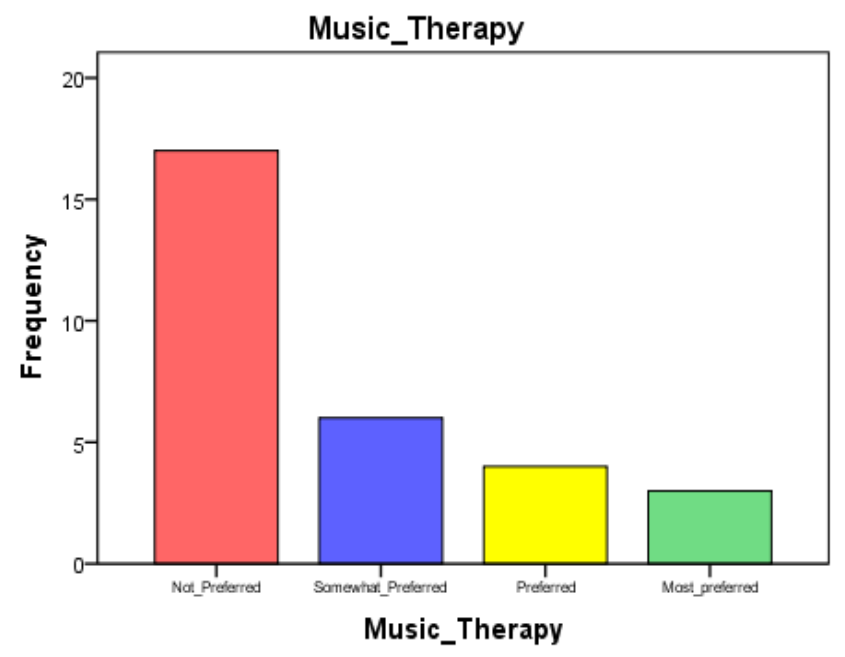

Figure 3: Participants' preferences for music therapy as a means of reducing pre-operative anxiety

Table 4 Summary of participants' rankings for counselling services as a means of reducing preoperative anxiety

\begin{tabular}{|l|c|c|c|}
\hline & Participants & Mean(SD) & 95\%Confidence Interval \\
\hline Totally not preferred & 13 & $2.00(1.11)$ & 1.33 to 2.67 \\
Not preferred & 9 & $2.00(1.11)$ & 1.14 to 2.86 \\
Preferred & 3 & $2.00(1.11)$ & -0.77 to 4.77 \\
Most preferred & 5 & $2.00(1.11)$ & -0.62 to3.38 \\
Total & $\mathbf{3 0}$ & & \\
\hline
\end{tabular}

Drug therapy is the most frequently used method of reducing pre-operative anxiety in virtually all hospitals. However, this method of reducing pre-operative anxiety is not a popular choice for the participants of this study. It can be seen from the participants' rankings that almost half of the participants $(13-43.3 \%, \mathrm{M}=2.00, \mathrm{SD}=1.11 ; 95 \% \mathrm{CI}=1.33$ to 2.67$)$ 'completely did not prefer' drug therapy as a means of reducing their anxiety before being operated on. Another nine $(30 \%)$ participants $(\mathrm{M}=2.00, \mathrm{SD}=1.11 ; 95 \% \mathrm{CI}=1.14$ to 2.86$)$ did 'not prefer' drug therapy to be used in reducing their pre-operative anxiety. On the other hand, only three participants $(10 \%, \mathrm{M}=2.00 \mathrm{SD}=1.11 ; 95 \% \mathrm{CI}$ to $0.77-4.77)$ preferred drug therapy and finally the remaining five participants $(16.7 \%, \mathrm{M}=2.00 \mathrm{SD}=1.11 ; 95 \% \mathrm{CI}=-0.62$ to 3.38$)$ ranked drug therapy as their most preferred intervention for reducing their pre-operative anxiety. The participants' scores for the preference of drug therapy are presented in the bar chart below.

Table 5: Participants' rankings for video film intervention as a means of reducing pre-operative anxiety

\begin{tabular}{|l|c|c|c|}
\hline \multicolumn{1}{|c|}{ Levels of preference } & & & 95\% Confidence Interval \\
\hline Totally not preferred & Participants & Mean (SD) & 1.39 to 4.12 \\
Not preferred & 5 & $2.70(1.06)$ & 1.74 to 3.68 \\
Preferred & 7 & $2.70(1.06)$ & 1.95 to 3.46 \\
Most preferred & 10 & $2.70(1.06)$ & 1.82 to 3.58 \\
Total & 30 & & \\
\hline
\end{tabular}


Video film is the intervention which the participants of this study have shown a high preference for. It can be seen from Table 5 that only five of the participants $(16.7 \%, \mathrm{M}=2.7 ; \mathrm{SD}=$ 1.06; CI [1.39 to 4.12]) ranked video film intervention as 'totally not preferred' intervention; seven (23.3\%, M=2.7; $\mathrm{SD}=1.06$; CI [1.74 to 3.68]) participants did 'not prefer' video film intervention as a method of anxiety reduction. Conversely, 10 participants $(33.3 \%, \mathrm{M}=2.7 ; \mathrm{SD}=$ 1.06; CI [1.95 to 3.46]) indicated that they preferred video film intervention and the remaining eight (26.7\%, M=2.7; SD=1.06; CI [1.82 to 3.58]) ranked video film intervention as their most preferred non-pharmaceutical preoperative anxiety reduction intervention to be used in reducing their pre-operative anxiety. Figure 4 presents the participants' levels of preference for video film intervention.

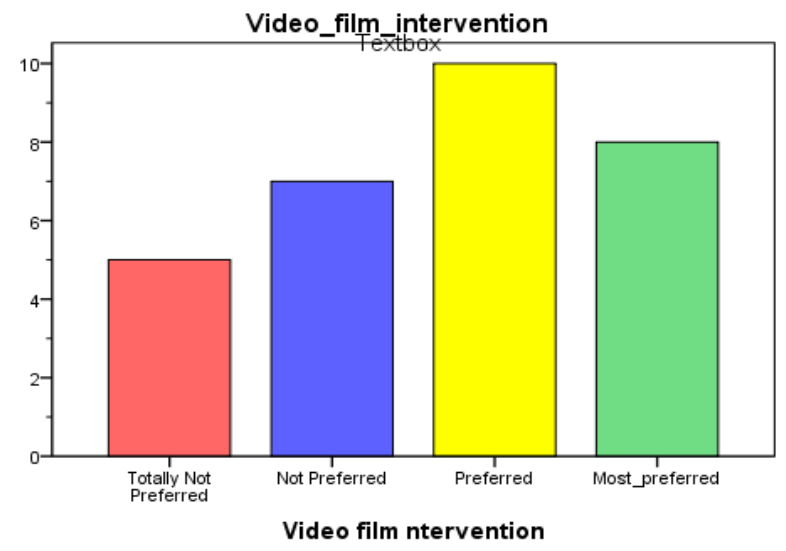

Figure 4: Participants' preferences for video film intervention as a means of reducing preoperative anxiety

\section{Gender difference in preference of non-pharmaceutical pre-operative anxiety reduction interventions}

To test the hypothesis that there is no significant difference between male and female elective surgical patients in preference of non-pharmaceutical pre-operative anxiety reduction interventions, an independent t-test was computed. The results obtained shows that there is no gender difference in preference of music intervention and video film intervention-music therapy $(M=2.08, S D=1.19) \mathrm{t}(28)=1.456, \mathrm{p}=.156$ level of significance. Video film intervention $(\mathrm{M}=3.00 \mathrm{SD}=.913) \mathrm{t}(28)=1.383, \mathrm{p}=1.78$ level of significance. However, gender difference was observed in counselling services $(M=2.31, S D=1.251) \mathrm{t}(28)=-0.253, \mathrm{p}=0.802$ level of significance; and information and education $(\mathrm{M}=2.69, \mathrm{SD}=1.032) \mathrm{t}(28)=-0.511, \mathrm{p}=0.61$ level of significance.

\section{DISCUSSION}

The present study is the first to assess elective surgical patients' preferences of nonpharmaceutical pre-operative anxiety reduction interventions. The findings reveal that there is a high preference for some and less preference of other non-pharmaceutical pre-operative anxiety reduction interventions among surgical patients undergoing elective surgery. The main finding is that more than half of the study participants $(66.7 \%)$ either 'preferred' or 'most preferred 'the information and education as a non-pharmaceutical intervention to be used in reducing their pre-operative anxiety before they undergo elective surgery. This is followed by another $60 \%$ of the participants who also 'preferred' or 'most preferred' video-film intervention as a means of pre-operative anxiety reduction before they undergo elective surgery. The third 'preferred' or 'most preferred' non-pharmaceutical pre-operative anxiety reduction intervention is counselling services in which $57 \%$ of the participants preferred it to be used on them in alleviating their anxiety before surgery. On the other hand, music therapy is the least preferred intervention where $76 \%$ of the participants reported they did 'not prefer' or 
'totally not prefer' music to be used in reducing their anxiety before undergoing surgery. The study participants did not significantly differ in terms of gender and preference of music intervention and video film interventions respectively. However, a statistically significant gender difference was found in their preference for counselling services and information and education interventions.

The difference in preference of non-pharmaceutical interventions found in this study is similar to what was reported in the literature by other researchers. For example, in this study, $67 \%$ of the participants 'preferred' or 'most preferred' information and education as an intervention to be used in reducing their pre-operative anxiety. A study by Wellala, Nanayakkara, De Silva, and Ponnamperuna (2012) reported that $72.5 \%$ of their participants desired information before the induction of anaesthesia and surgery and that more female $(81.93 \%)$ than male $(65.5 \%)$ patients desired information. Even though the present study also used surgical patients as participants and found similar results to the study by Wellala et al., the two studies differ in their methodology. Wellala et al. used 200 participants as opposed to the 30 participants used in this study. Also, the method of data collection differs with Wellala using a questionnaire administered survey; the current study utilised a systematic preference elicitation, using the ranking method.

On video film intervention, some randomised controlled trials (Arabul et al., 2013; Jlala, French, Foxall, Hardman, \& Bedforth, 2010; Wu et al., 2014) found that participants assigned to the video film intervention group were more satisfied with the video film interventions and that they had lower post-operative STAI scores as opposed to participants in the control groups. These findings suggest that pre-operative video intervention increases the patients' satisfaction with the cardiac catheterisation procedure as well as upper and lower limb surgeries respectively. In the present study, though the focus is to find the preference of nonpharmaceutical pre-operative anxiety reduction interventions from elective surgical patients, the finding that $70 \%$ of the participants 'preferred' or 'most preferred' video film as a preoperative anxiety reduction intervention means that, when video film intervention is administered to the participants of this study, with the aim of reducing their pre-operative anxiety, they stand a chance of responding positively and have their pre-operative anxiety reduced.

Many studies have been conducted to test the effectiveness of music intervention in reducing pre-operative anxiety. As with video film intervention, most of the studies used randomised controlled trials to collect information from their participants. For example, (Lee, Chao, Yiin, Chiang, \& Chao, 2011; Lee et al., 2012; Lin, Lin, Huang, Hsu, \& Lin, 2011; Ni, Tsai, Lee, Kao, \& Chen, 2012; Weeks \& Nilsson, 2011) have all conducted randomised controlled trials to establish the efficacy of music therapy in reducing pre-operative anxiety. The outcomes of these studies show that music intervention significantly reduces pre-operative anxiety in the participants randomised to a music group compared to those assigned to only usual care or no music groups. However, despite the reported evidence of the efficacy of music intervention in the aforementioned studies, music therapy remains an unpopular choice among the participants of this study. Of all the non-pharmaceutical pre-operative anxiety reduction interventions presented to the participants of this study, music therapy is the least preferred. Only $23.3 \%$ of the participants express a preference for music to be used in reducing their preoperative anxiety. The large majority-76.7\%- does not like music as an intervention to be used in reducing their anxiety prior to surgery. This is attributed to the participants' cultural and religious beliefs. The position of music in Islam is what accounted for the low preference ranking of music intervention by the participants of the study. In the Muslim-dominated part of northern Nigeria, and particularly among adherents of the tariqatu-Tijjaniyya and the Sunni 
Islamic sects, playing or listening to music is a taboo. However, adherents of other Islamic sects do not share this view. Consequently, among the participants of the study who hold negative beliefs about music, playing music to reduce their pre-operative anxiety is not acceptable. According to these participants, rather than listening to music before going for surgery, it is better to listen to a recitation of the Qur'an which also has a rhythm, like music. They believe that when reading the Holy Qur'an or listening to it, shaitans the person is surrounded by the angels of Rahma (blessing). But for a person listening to music, he/she is surrounded by (devils). This view about music is only held by participants of the Muslim faith. However, for patients of the Christian faith, which has no restrictions on playing and listening to music, they find music therapy as a relevant and acceptable intervention.

\section{LIMITATIONS OF THE STUDY}

Apart from the findings that participants have preferences for some and no preference for other non-pharmaceutical pre-operative anxiety reduction interventions, and that a significant gender difference exists in the preferences of these interventions, the findings have to be treated with caution as the study is not without limitations. First, the study is a single centre study which is naturally and relatively easy to conduct and makes the findings not inadequate to inform clinical practice. However, given the fact that no study of elective surgical patients' preferences of non-pharmaceutical pre-operative anxiety reduction interventions has previously been conducted, the present study will provide preliminary empirical data that will form the bases for conducting large-scale multicentre studies, the results of which may inform clinical practice. The study also used a small number of participants. This may increase the probability of a 'Type II error', which also reduces the generalisability of the findings of the study. However, considering the nature of the study population-elective surgical patients, and the hospital setting where the study was conducted, the use of 30 participants is sufficient to provide significant results. That data for this study were collected by means of a systematic approach, using a ranking procedure. This method was chosen to be used bearing in mind the educational status of the population from which the participants were drawn. There is a need to further improve the method by using validated and standardised preference elicitation instruments. More so, the use of the interview method will also help provide qualitative data that may expose reasons why patients preferred the intervention they prefer and why they did not prefer what they do not prefer. Although the participants of the study have shown their preference of the non-pharmaceutical preoperative anxiety reduction interventions, it is not known whether the clinicians would use them in practice. There is a need therefore to assess the clinicians' views about and their acceptance of these non-pharmaceutical preoperative anxiety reductions interventions and to find out if they would use them on their patients in their clinical practice.

\section{CONCLUSION}

Although it is the widely advocated that patient preferences be considered in healthcare treatment decisions, knowledge on this subject matter is very scanty. The few available information is related to patients' preference of one or more pharmaceutical intervention(s) over another. No data is available on patients' preference of non-pharmaceutical interventions for alleviating anxiety related to surgery. Findings of this study shows that, anxious surgical patients have different preferences for preoperative anxiety reduction interventions. Thus, clinicians should be aware that those patients' needs might not be the same with theirs and that they cannot be discerned from the patients without engaging with them in an atmosphere that is relaxed and conducive for them to explain. Clinicians providing intervention to anxious preoperative patients are therefore encouraged to provide such a conducive environment and provide the patients with variety of intervention approaches to select from. By so doing, 
interventions appropriate to the patients' needs will be provided to achieve a better postoperative outcome.

\section{References}

Ali, S., \& Ronaldson, S. (2012). Ordinal preference elicitation methods in health economics and health services research: using discrete choice experiments and ranking methods. British medical bulletin, 103(1), 21-44.

Arabul, M., Kandemir, A., Celik, M., Torun, S., Beyazit, Y., Alper, E., . . Ünsal, B. (2013). Impact of video information before unsedated upper gastrointestinal endoscopy on patient satisfaction and anxiety: a prospective randomized trial. PRZEGLAD GASTROENTEROLOGICZNY, 8(1), 44-49.

Collins, S., Britten, N., \& Ruusuvuori, J. (2007). Patient Participation In Health Care Consultations: Qualitative Perspectives: Qualitative Perspectives: McGraw-Hill Education (UK).

Coulter, A. (2011). Engaging patients in healthcare: McGraw-Hill Education (UK).

Coulter, A., Parsons, S., \& Askham, J. (2008). Where are the patients in decision-making about their own care. World Health Organization.

de Bekker-Grob, E. W., Ryan, M., \& Gerard, K. (2012). Discrete choice experiments in health economics: a review of the literature. Health Economics, 21(2), 145-172.

Fok, D., Paap, R., \& Van Dijk, B. (2012). A Rank-Ordered Logit Model With Unobserved Heterogeneity In Ranking Capabilities. Journal of applied econometrics, 27(5), 831-846.

Gutknecht, M., Schaarschmidt, M. L., Herrlein, O., Augustin, M. J. J. o. t. E. A. o. D., \& Venereology. (2016). A systematic review on methods used to evaluate patient preferences in psoriasis treatments. 30(9), 1454-1464.

Ha, J. F., \& Longnecker, N. (2010). Doctor-patient communication: a review. The Ochsner Journal, 10(1), 38-43.

Jlala, H., French, J., Foxall, G., Hardman, J., \& Bedforth, N. (2010). Effect of preoperative multimedia information on perioperative anxiety in patients undergoing procedures under regional anaesthesia. British journal of anaesthesia, 104(3), 369-374.

Kocsis, J. H., Leon, A. C., Markowitz, J. C., Manber, R., Arnow, B., Klein, D. N., \& Thase, M. E. (2009). Patient preference as a moderator of outcome for chronic forms of major depressive disorder treated with nefazodone, cognitive behavioral analysis system of psychotherapy, or their combination. The Journal of clinical psychiatry.

Lee, K.-C., Chao, Y.-H., Yiin, J.-J., Chiang, P.-Y., \& Chao, Y.-F. (2011). Effectiveness of different music-playing devices for reducing preoperative anxiety: a clinical control study. International journal of nursing studies, 48(10), 11801187.

Lee, K.-C., Chao, Y.-H., Yiin, J.-J., Hsieh, H.-Y., Dai, W.-J., \& Chao, Y.-F. (2012). Evidence that music listening reduces preoperative patients' anxiety. Biological research for nursing, 14(1), 78-84.

Lin, P.-C., Lin, M.-L., Huang, L.-C., Hsu, H.-C., \& Lin, C.-C. (2011). Music therapy for patients receiving spine surgery. Journal of clinical nursing, 20(7/8), 960-968. doi:10.1111/j.1365-2702.2010.03452.x

McHugh, R. K., Whitton, S. W., Peckham, A. D., Welge, J. A., \& Otto, M. W. (2013). Patient preference for psychological vs. pharmacological treatment of psychiatric disorders: a meta-analytic review. The Journal of clinical psychiatry, 74(6), 595.

Miranda, J. (2009). An exploration of participants' treatment preferences in a partial RCT. CJNR (Canadian Journal of Nursing Research), 41(1), 276-290.

Mitchell, P., Wynia, M., Golden, R., McNellis, B., Okun, S., Webb, C. E., .. Von Kohorn, I. (2012). Core principles \& values of effective team-based health care: Institute of Medicine Washington, DC.

Montori, V. M., Brito, J. P., \& Murad, M. H. (2013). The optimal practice of evidence-based medicine: incorporating patient preferences in practice guidelines. Jama, 310(23), 2503-2504.

Ni, C. H., Tsai, W. H., Lee, L. M., Kao, C. C., \& Chen, Y. C. (2012). Minimising preoperative anxiety with music for day surgery patients-a randomised clinical trial. Journal of clinical nursing, 21(5-6), 620-625.

Pomey, M.-P., Ghadiri, D. P., Karazivan, P., Fernandez, N., \& Clavel, N. (2015). Patients as partners: a qualitative study of patients' engagement in their health care. PLoS ONE, 10(4), e0122499.

Ratcliffe, J., Brazier, J., Tsuchiya, A., Symonds, T., \& Brown, M. (2009). Using DCE and ranking data to estimate cardinal values for health states for deriving a preference-based single index from the sexual quality of life questionnaire. Health Economics, 18(11), 1261-1276. 
Ryan, M., \& Farrar, S. J. B. B. M. J. (2000). Using conjoint analysis to elicit preferences for health care. 320(7248), 1530.

Siminoff, L. A. (2013). Incorporating patient and family preferences into evidence-based medicine. $B M C$ medical informatics and decision making, 13(3), S6.

Swift, J. K., Callahan, J. L., \& Vollmer, B. M. (2011). Preferences. Journal Of Clinical Psychology, 67(2), $155-165$.

Tariman, J. D., Berry, D. L., Cochrane, B., Doorenbos, A., \& Schepp, K. (2012). Physician, patient and contextual factors affecting treatment decisions in older adults with cancer: a literature review. Paper presented at the Oncology Nursing Forum.

Vahdat, S., Hamzehgardeshi, L., Hessam, S., \& Hamzehgardeshi, Z. (2014). Patient involvement in health care decision making: a review. Iranian Red Crescent Medical Journal, 16(1).

Weeks, B. P., \& Nilsson, U. (2011). Music interventions in patients during coronary angiographic procedures: A randomized controlled study of the effect on patients' anxiety and well-being. European Journal of Cardiovascular Nursing, 10(2), 88-93.

Weernink, M. G., Janus, S. I., Van Til, J. A., Raisch, D. W., Van Manen, J. G., \& IJzerman, M. J. J. P. m. (2014). A systematic review to identify the use of preference elicitation methods in healthcare decision making. 28(4), 175185.

Wellala, K., Nanayakkara, A., De Silva, A., \& Ponnamperuna, A. (2012). Descriptive study on pre-anaesthetic information desired by patients who undergo surgery. Sri Lankan Journal of Anaesthesiology, 20(1).

Wu, K.-L., Chen, S.-R., Ko, W.-C., Kuo, S.-Y., Chen, P.-L., Su, H.-F., \& Chang, W.-Y. (2014). The effectiveness of an accessibility-enhanced multimedia informational educational programme in reducing anxiety and increasing satisfaction of patients undergoing cardiac catheterisation. Journal of clinical nursing, 23(13/14), 2063-2073. doi:10.1111/jocn.12469 